\title{
Exacerbations of chronic obstructive pulmonary disease
}

J Kidney, T McManus, P V Coyle

Respiratory diseases are the most common cause of death in the UK but, while targets are set for every other major disease category, lung disea ses do not get a mention.

$\mathrm{R}$ espiratory diseases are the most common cause of death with a $16 \%$ higher mortality rate than with coronary heart disease. Chronic obstructive pulmonary disease (COPD) is the most common chronic respiratory illness. At the end of its long course over 30000 people die from COPD each year in the UK. It is a disease of the poor, with a death rate in men of working age 14 times higher in unskilled manual workers than in professionals. ${ }^{1}$

The burden to the health service is enormous. In the UK acute respiratory admissions account for $25 \%$ of all emergency medical admissions ${ }^{2}$ and cost $£ 1.1$ billion. ${ }^{3}$ COPD exacerbations account for more than half of these admissions, ${ }^{2}$ so are likely to cost in excess of $£ 500$ million per annum. In planning for service delivery, COPD admissions throw a spanner in the works. During the summer there is a steady number of admissions but in winter this rises by up to threefold (un published data, M ater Hospital). This is the time of year when images of patients waiting on trolleys in $A \& E$ departments fill our television screens. The domino effect of admissions with COPD goes beyond A\&E departments; beds in medical wards are filled to capacity, beds in surgical wards are filled with medical patients, surgical patients are turned away with their operations cancelled, and politically sensitive waiting list targets are not met.

Respiratory diseases are not on government targets for action. Breathlessness and the most common cause of death is hardly on the political radar. This is only going to get worse. Deaths from COPD are set to rise from the sixth to the third most common cause of death by $2020 .{ }^{4}$

ROLE OF BACTERIAL INFECTION

The East London COPD study has done much to help our understanding of the condition. In this edition of Thorax Patel and colleagues have reported another aspect of their study group. They examined the sputum for the presence of bacteria and compared colonised and noncolonised subjects for the frequency of COPD exacerbations. ${ }^{5}$ They divided the group into frequent and infrequent exacerbators and found that patients who had pathogens in their sputum were more likely to be frequent exacerbators with an odds ratio of 6.25 . They also found that there was an increased level of interleukin-8 (IL-8) in the sputum of the frequent exacerbators, but this just failed to reach statistical significance. However, the levels of IL-8 correlated with the bacterial count.

$$
\begin{gathered}
\text { "[CO PD }] \text {... will continue } \\
\ldots \text { as the "Cinderella" of } \\
\text { medicine" }
\end{gathered}
$$

The study design was such that the sputum assay was obtained in the middle of the study period and exacerbations were identified from diary cards before and after the sputum assay. As such it is not a prospective study. While the East London COPD study was ongoing, others have shown that more than a quarter of patients with a primary care diagnosis of COPD have CT evidence of bronchiectasis. ${ }^{6}$ This is an area of significant interest because it will change future investigation and treatment strategies in patients with COPD. It is, however, beyond the scope of this current study to determine the extent of bronchiectasis in their population.

\section{ROLE OF VIRUS INFECTION}

In previous work published from the East London COPD study Seemungal et al found that one third of exacerbations of COPD were associated with common respiratory viruses, using molecular techniques. ${ }^{7}$ Similar findings were reported using traditional virological methods on a mixed population of patients with COPD and heart failure. ${ }^{8}$ The principal advantages of molecular techniques are their increased sensitivity and higher detection rate of rhinoviruses which can be difficult to culture or identify serologically.

Epidemic viruses may not be the only viruses involved in COPD. Hogg's group in Vancouver have identified the presence of adenovirus E1A early antigens in patients with COPD. ${ }^{9}$ Infection early in life may lead to latent infection which may be important in priming cells for a subsequent role in the development of COPD. Their findings indicate that EIA continues to be expressed within the epithelial cell and, given the right circumstances such as smoke exposure, COPD will result.

A role for viral infection in the pathogenesis of COPD has been supported by the finding of raised titres to common respiratory viruses (CM V and adenoviruses) in a Norwegian population with COPD. ${ }^{10}$ By comparing umbilical cord blood samples at birth and heel prick samples, we have confirmed that infection with adenovirus is the commonest respiratory virus in early infancy, with up to $23 \%$ of children undergoing seroconversion by 6 months of age. ${ }^{11}$ In an elaborate study by Gilmour \& al a cultured epithelial cell line (A549) was transfected with adenovirus E1A gene and exposed to pollution particles ( $\left.\mathrm{PM}_{10}\right)$ resulting in a significant rise in IL-8. ${ }^{12}$

\section{MECHANISMS OF DISEASE}

Patel et $\mathrm{al}^{5}$ identified a correlation be tween IL-8 levels and bacterial count. IL-8 is secreted by epithelial cells and causes neutrophils to transmigrate the airway epithelium. The principal mechanism of this process is via intercellular adhesion molecule 1 (ICAM-1) on the epithelium which binds to the counter ligand CD18/CD11b on the neutrophil. This process can be enhanced by the addition of tumour necrosis factor $\alpha$ (TNF $\alpha$ ) and interferon $\gamma$ (IFN $\gamma$ ) to epithelial cells in vitro. ${ }^{13}$

Bacterial colonisation of the airways is associated with increased levels of $\mathrm{TNF} \alpha,{ }^{14}$ and infection with viruses causes a rise in lymphocyte derived IFN $\gamma$. Both of these chemokines significantly increase epithelial ICAM -1, thereby compounding the effect of IL-8 in enhancing neutrophil accumulation in the airways. Furthermore, ICAM-1 is the major surface receptor for rhinovirus infecting epithelial cells, ${ }^{15}$ and rhinovirus is the most common viral infection in COPD exacerbations. ${ }^{7}$ This sets the scene for a vicious circle of increased adhesion of viruses, increased cytokine expression, and increased neutrophil accumulation in the airway.

While each step in this inflammatory "catch 22" is established, we can only await pharmaceutical development and trials to try to break the cycle. Until that time we have to watch as COPD increases year on year and is expected to rise from its current sixth to the third most common cause of death in $2020 .{ }^{4}$ We also have to watch as targets are set for every other major disease category, yet lung diseases which kill most people do not get a mention. COPD will continue to fill 
the A\&E departments, shut the hospitals, close the operating theatres due to a lack of beds, and break every target in every acute hospital service. I ronically, it will continue its lonely position as the "Cinderella" of medicine.

Thorax 2002;57:753-754

\section{Authors' affiliations}

J Kidney, T McManus, Department of

Respiratory M edicine, M ater Hospital Trust, Belfast, UK

TMCManus, P V Coyle, Regional Virology

Laboratory, Royal Victoria Hospital, Belfast, UK

Correspondence to: Dr J Kidney, Department of Respiratory Medicine, M ater Hospital, C rumlin Road, Belfast BT14 6AB, UK;

joekidney@utvinternet.com

\section{REFERENCES}

1 British Thoracic Society. The burden of lung disease. London: British Thoracic Society, 2001
2 Anderson HR, Esmail A, Hollowell I, et al. Epidemiologically based needs assessment: lower respiratory disease. London: Department of Health, 1994.

3 Department of Health. A nnual trust returns. London: Department of Health, 1999.

4 Lopez AD, M urray CL. The global burden of disease. $N$ ature Med 1998;4:1241-3

5 Patel IS, Seemungal TAR, W ilks M, et al. Relationship between bacterial colonisation and the frequency, character and severity of CO PD exacerbations. Thorax 2002.57:759-64

6 O'Brien C, G uest PJ, Hill SL, et al. Physiological and radiological characterisation of patients diagnosed with chronic obstructive pulmonary disease in primary care. Thorax 2000:55:635-42.

7 Seemungal TA, Harper-0 wen R, Bhow mik A et al. Symptoms, inflammatory markers and respiratory viruses in acute exacerbations and stable CO PD. Am J Respir Crit Care Med 2001;164:1618-23.

8 Walsh EE, Falsey AR, Hennessy PA. RSV and other virus infections in persons with chronic cardiopulmonary disease. Am J Respir Crit Care Med 1999;160:791-5

9 Hogg JC. Childhood viral infection and the pathogenesis of asthma and chronic obstructive lung disease. Am J Respir Crit Care Med 1999;160:26-8.
10 Omenaas E, Bakke P, Eide GE, et al. Serum respiratory virus antibodies: predictor of reduced one-second forced expiratory volume (FEV1) in N orwegian adults. Int J Epidemiol 1996;25:134-41.

11 Nelson JK, Sheils MD, Stewart M C et al. Seroprevalence of respiratory virus infections in an infant population with a multiantigen fluorescence immunoassay using heel-prick blood samples collected on filter paper. Pediatr Res 1999;45:799-802.

12 Gilmour PS, Rahman I, Hayashi S, et al. Adenoviral E1A primes alveolar epithelia cells to PM (10)-induced transcription of interleukin-8. Am J Physiol Lung Cell Mol Physiol 2001;281:L598-606.

13 Kidney JC, Proud D. N eutrophil

transmigration across human airway epithelial monolayers: mechanism and dependence on electrical resistance. Am J Respir Cell Mol Biol 2000;23:389-95.

14 Soler N, Ewig S, Torres A, et al. Airway inflammation and bronchial microbial patterns in patients with stable chronic obstructive pulmonary disease. Eur Respir J 1999;14:1015-22

15 Staunton DE, M erluzzi VJ, Rothlein R, et al. A cell adhesion molecule, ICAM-1, is the major surface receptor for rhinoviruses. Cell 1989;56:849-53.

\title{
G ene expression profiling: good housekeeping and a clean message
}

\author{
R C Chambers
}

\section{M icroarray technology offers us the means of monitoring gene expression on a scale which was hard to envisage only a few years ago.}

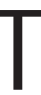
here is no doubt that gene expression studies based on evaluating mRNA levels for single or multiple genes of interest in human lung biopsy tissue have had a major impact on our understanding of the molecular mechanisms underlying respiratory disease. The recent advent of microarray technology has added further impetus to the central paradigm that mRNA quantification in lung tissue can shed light on pathogenesis and identify new targets for therapeutic intervention. This technology is now so advanced that it allows the parallel monitoring of entire ge nomes using microarrays with a surface area equivalent to just a few square centimetres and as little as $5 \mu \mathrm{g}$ RNA starting material.

Since its first application in the mid $1990 \mathrm{~s},{ }^{1}$ microarray technology has been applied to all aspects of biomedical research with over 60 papers in respiratory research alone. It has been successfully used for the classification and molecular diagnosis of lung cancer, ${ }^{2}$ the identification of potential target genes for therapeutic intervention in idiopathic pulmonary fibrosis, ${ }^{3}$ mechanistic studies in animal models of asthma ${ }^{4}$ and pulmonary fibrosis, ${ }^{5}$ and for profiling lung development. ${ }^{6}$ Global expression profiling of cellular responses in vitro has provided new insights into the transcriptional programs involved in cytokine signalling, ${ }^{7}$ growth arrest and apoptosis, ${ }^{8}$ and it is already enabling us to understand the operation of functional gene networks.

\section{MICROARRAY PLATFORMS}

Although a number of microarray platforms have been devel oped, microarrays come in two basic formats. Complementary DNA (cDNA) arrays usually contain polymerase chain reaction (PCR) products generated from cDNA libraries or clone collections, spotted onto glass slides or nylon membranes. Expression values are based on the competitive hybridisation of two samples being directly compared following the incorporation of two fluorescent dyes ( Сy3 and Cy5) on a single array. In contrast, oligonucleotide arrays (for example, Affymetrix GeneChips) contain relatively short sequences (20-mers) synthesised onto silicon wafers in situ by photolithography or arrayed as pre-synthesised oligonucleotides onto glass slides. The final target consists of biotin labelled cRNA and each sample is hybridised to a separate array. Hybridisation is detected by staining with a streptavidinphyocoerythrin conjugate followed by confocal fluorescencelaser scanning. The advantage of oligonucleotide arrays is that they contain multiple validated probe sequences for each gene and mismatch control sequences to allow correction of non-specific hybridisation signals. In contrast, cDNA arrays usually consist of user defined probe sequences but allow a much greater degree of flexibility and are generally cheaper as slide printing can be performed in house.

\section{EXTRACTING BIOLOGICAL MEANING}

$M$ anaging and mining the huge amount of data generated by microarray experiments remains a major challenge for most users. Although this side of microarray analysis is still considered a major bottleneck, help is at hand via a plethora of online data mining, clustering, and analysis tools. In fact, most of the best tools are available to academic users as freeware upon request. A detailed description of these tools is beyond the scope of this editorial. However, Gene Express (http://www.thoracic.org/ geneexpress/), a new column edited by Naftali Kaminski and hosted by the ATS website, is a valuable resource aimed specifically at lung researchers and an excellent route to other sites of interest.

Despite its growing use in both academia and industry, microarray experiments are still considered by many 
as nothing more than a sophisticated fishing trip. This is because microarray analysis challenges the traditional hypothesis driven method of investigation and shifts the emphasis towards hypothesis generation. Investigators are then faced with what is probably the greatest challenge-namely, the extraction of biological meaning from microarray data and the prioritisation of candidate genes for follow up. Faced with hundreds of possibilities, it is not surprising that investigators have, in the past, tended to focus on genes they recognise and can integrate into a reasonable hypothesis regarding their likely role in the disease process. Fortunately, the need to address these limitations of microarray analysis is fuelling the rapid development of novel computational tools. This includes unbiased scoring methods for identifying the most meaningful and informative genes in microarray experiments. One such tool has recently been applied to great effect to funnel and prioritise candidate genes for follow up in expression studies of human lung biopsy material from patients with pulmonary fibrosis. ${ }^{3}$ Used in combination with computational tools which allow the visualisation of gene expression data on maps representing biological pathways (for example, GenM APP at http://gladstonegenome.ucsf.edu/) and programs based on artificial neural networks which can be trained to recognise signature expression profiles, ${ }^{9}$ these tools are likely to significantly accelerate our understanding of the molecular basis of disease.

\section{VALIDATION OF MICROARRAY DATA}

Although microarray technology is improving rapidly and confidence in the data generated is growing, validation of microarray expression trends using a second readout remains a critical requirement. This is especially important if the sample size is too small to allow rigorous statistical analysis. For this purpose, the real time fluorescence based reverse transcriptase polymerase chain reaction (RT-PCR) is generally the method of choice. However, in this issue of Thorax, Glare $\& \mathrm{al}^{10}$ revisit one of the most stubborn problems associated with all RT-PCR based methods-namely, the choice of a reference gene with which to normalise signals obtained to allow the legitimate comparison between samples and eliminate differences of nonbiological origin. One of the most commonly used methods is to normalise against a housekeeping gene because its mRNA levels are thought to remain constant. Using competitive RT-PCR, Glare et al provide compelling evidence that mRNA levels of two of the most commonly used housekeeping genes in asthmatic airways-glyceraldehyde-3phosphate dehydrogenase (GAPDH) and $\beta$-actin-are, in fact, highly variable and therefore totally unsuitable for normal ising the expression levels of potential genes of interest. This study is a wel come addition to a growing body of evidence that mRNA levels of a number of traditional housekeeping genes are not invariable under a variety of experimental and pathological conditions. ${ }^{11}$ The evidence is now so strong for samples obtained in vivo that their use should either be discontinued or can only be viewed as valid when appropriate experiments have been performed to confirm that their expression is indeed constant under the experimental conditions of the study.

So what are the alternatives for normalising gene expression data? There are no ideal solutions but, for conventional gene expression studies, the use of total cellular RNA has been proposed as one of the least unreliable methods for data normalisation. ${ }^{12}$ Although the use of total RNA levels for normalising expression data derived from patient material still has to be fully validated, recent technical advances for RNA quantitation-including the RiboGreen RNA quantification assay and the Agilent Bioanalyzer which allows RNA quantity and quality assessment in a single step-are likely to prove very useful for studies of human biopsy material with very low RNA yields. Another alternative is to use ribosomal RNA (rRNA) which makes up the bulk of total RNA. Despite reservations regarding changes in expression levels and potential imbalances in rRNA and mRNA fractions between different samples, 18S rRNA has recently been validated for normalising expression levels by quantitative RT-PCR analysis under a number of experimental conditions and is demonstrably more reliable than normalising to housekeeping genes. ${ }^{11}{ }^{13}$ While considering the issue of normalisation, it is also worth pointing out that, regardless of the platform used, uncertainties relating to the use of housekeeping genes for signal normalisation are al so relevant to microarray experiments. For oligonucleotide based arrays, the most commonly used approach is to scale or normalise the output data using a transcriptome equivalent strategy (or global normalisation) in order to derive an average intensity for each array with the assumption that the total sum of all transcripts present is similar between samples.

Finally, it is also worth remembering that gene expression studies measure mRNA levels and no more. Since most genes are also highly regulated at the post-transcriptional stage, changes in mRNA levels may not necessarily reflect changes at the protein level. In addition, interpreting expression studies in disease versus control tissue is often confounded by the very dramatic differences in cell populations present within the two types of tissue. Genes which appear to be highly differentially expressed may therefore reflect changes in the cellular composition of the tissue rather than changes in gene expression per se. Additional analysis by conventional immunohistochemistry and/or in situ hybridisation therefore becomes essential when analysing whole biopsy tissue. Similarly, important changes in gene expression may be masked because of dilution of the message. This may be particularly problematic when dealing with biopsy tissue where the disease is confined to a small number of cells within the sample. Recent advances in RNA amplification technology ${ }^{14}$ and laser capture microdissection (LCM) to sample individual cell populations within a biopsy sample are proving particularly useful for addressing these potential problems. ${ }^{2}$

In conclusion, we now have the means of monitoring gene expression on a scale which was hard to envisage only 5 years ago. The integration of this technology with rapidly evolving innovations in novel computational tools, public domain data repositories, in combination with the appropriate post-microarray validation experiments is likely to have a major impact on our understanding of complex human disease processes in the future.

Thorax 2002;57:754-756

Correspondence to: $\mathrm{Dr} \mathrm{R} \mathrm{C}$ Chambers, Centre for Respiratory Research, University College London, London W C 1E 6JJ, UK

R.Chambers@ucl.ac.uk

\section{REFERENCES}

1 Schena M, Shalon D, Davis RW, et al. $Q$ uantitative monitoring of gene expression patterns with a complementary DN A microarray. Science 1995;270:467-70.

2 Miura K, Bowman ED, Simon R, et al. Laser capture microdissection and microarray expression analysis of lung adenocarcinoma reveals tobacco smoking- and prognosis-related molecular profiles. Cancer Res 2002;62:3244-50.

3 Zuo F, Kaminski N, Eugui E, et al. G ene expression analysis reveals matrilysin as a key regulator of pulmonary fibrosis in mice and humans. Proc $N$ atl Acad Sci USA 2002;99:6292-7.

4 Zou J, Young S, Zhu F, et al. Microarray profile of differentially expressed genes in a monkey model of allergic asthma. G enome Biol 2002; 3:research0020.

5 Kaminski N, Allard JD, Pittet JF, et al. G lobal analysis of gene expression in pulmonary fibrosis reveals distinct programs regulating lung inflammation and fibrosis. Proc $\mathrm{Nat}$ Acad Sci USA 2000;97:1778-83

6 Mariani TJ, Reed JJ, Shapiro SD. Expression profiling of the developing mouse lung: insights into the establishment of the extracellular matrix. Am J Respir Cell Mol Biol 2002;26:541-8

7 Lee JH, Kaminski N, Dolganov G, et al. Interleukin-13 induces dramatically different transcriptional programs in three human airway cell types. Am J Respir Cell Mol Biol 2001;25:474-85.

8 Kannan K, Kaminski N, Rechavi G, et al. DN A microarray analysis of genes involved in p5 3 mediated apoptosis: activation of Apaf-1. Oncogene 2001;20:3449-55 
9 Khan J, W ei JS, Ringner M, et al. Classification and diagnostic prediction of cancers using gene expression profiling and artificial neural networks. $N$ ature $M$ ed 2001;7:673-9.

10 Glare EM, Divjak M, Bailey MJ, et al. B-Actin and GAPDH housekeeping gene expression in asthmatic airways is variable and not suitable for normalising mRN A levels. Thorax 2002;57:765-70.
11 Goidin D, Mamessier A, Staquet MJ, et al. Ribosomal 18S RN A prevails over glyceraldehyde-3-phosphate dehydrogenase and beta-actin genes as internal standard for quantitative comparison of mRN A levels in invasive and noninvasive human melanoma cell subpopulations. Anal Biochem 2001;295:17-21.

12 Bustin SA. Absolute quantification of mRN A using real-time reverse transcription polymerase chain reaction assays. I $\mathrm{MOl}$ Endocrinol 2000:25:169-93.

13 Schmittgen TD, Zakrajsek BA. Effect of experimental treatment on housekeeping gene expression: validation by real-time, quantitative RT-PCR. I Biochem Biophys Methods 2000;46:69-81.

14 Wang E, Miller LD, O hnmacht GA, et al. High-fidelity mRN A amplification for gene profiling. N ature Biotechnol 2000;18:457-9.

\section{Psychological consequences of segregation resulting from chronic Burkholderia cepacia infection in adults with $C F$}

\section{A J A Duff}

Patients with CF segregated because of Burkholderia cepacia infection must be helped to assemble coherent structures for social relationships if they are to adapt successfully to such management.

n 1997 the median life expectancy for individuals with cystic fibrosis (CF) was 31.5 years in the UK ${ }^{1}$ and it has been suggested that those born today can expect to live well into their mid 40 s. $^{2}$ However, there is huge variability in the physical condition of adults with CF. While malabsorption, osteoporosis, diabetes, and liver failure all contribute to incapacity, lung disease is the main cause of morbidity and mortality. Some patients have near normal levels of lung function. Others, however, are debilitated by dyspnoea and dependent on oxygen.

Lung disease in CF is primarily due to the consequences of infection. In the first decade of life Staphylococcus aureus and Haemophilus influenzae are the predominant organisms in sputum, while in older children and adults Pseudomonas aeruginosa is most common. ${ }^{3}$ In the past 15 years some CF centres have had epidemics of Burkholderia cepacia infection. Although patients respond to standard antibiotic treatment, ${ }^{4}$ most become chronically infected and experience a more rapid decline in lung function. The reasons for this are still unclear, although recent microbiological findings suggest that there are different pathogenic potentials of various B cepacia genomovars. ${ }^{5}$ In the UK prevalence rates vary between centres but increase significantly if spread from patient to patient is not prevented. In this respect, B cepacia differs from other bacteria in that it is usually caught through close or frequent contact with another B cepacia positive CF patient. ${ }^{56}$

In most UK adult CF centres it is now accepted practice to separate patients who are infected with B cepacia from those who are not. Guidelines on cross infection effectively mean managing infected patients in isolation, away from the main CF wards, but even this may not be sufficient to prevent the spread of the organism. Contemporary advice to patients extends segregation to outside hospitalsdirecting them not to attend CF meetings, not to have any physical contact with $B$ cepacia negative CF patients, and to adopt impeccable hygienic behaviour. ${ }^{7}$ Although difficult, where this has been done fewer patients with CF become infected with $B$ cepacia for the first time ${ }^{8}$ and some clinicians now report a decrease in the overall number of cases. However, as a result of the emergence of cross infection in CF patients by a multiresistant strain of $P$ aeruginosa, some clinicians now advocate segregating patients according to their microbiological status. ${ }^{90}$ Others have questioned the wisdom of adopting such practice, highlighting - in addition to clinical reasons-the potential emotional impact on patients and their families. ${ }^{11}$

\section{PSYCHOLOGICAL CONSEQUEN CES OF SEGREGATION}

While it is known that adults with chronic illness are at a greater risk of developing psychological problems, ${ }^{12}$ prevalence rates of such difficulties in adult CF populations are still largely unknown. What is known is that individuals at the end stages of the condition are even more vulnerable to psychological problems resulting from increasing "loss" of, for example, mobility, autonomy, relationships, and quality of life. Furthermore, the disease becomes more "visible" and intrusive and less predictable, which can give rise to feelings of anhedonia, helplessness, fear, and anxiety. ${ }^{13}$ With this in mind, while much has been published on the physical benefits of segregating patients with CF, almost no information exists on the psychosocial consequences of such practices.

It is well recognised that being "hospitalised" has a negative effect on psychological functioning. ${ }^{14-17}$ I solation in hospital has the potential to have even greater negative effects on emotional well being. One study reported that over $42 \%$ of patients identified negative emotions associated with isolation. ${ }^{18}$ Such patients have significantly higher rates of anxiety and depression and significantly lower levels of self-esteem and control. ${ }^{19}$ Evidence from other patient groups who have experienced segregated and isolating medical treatments (such as those with cancer, leprosy or HIV positive patients) suggests that the experience is confining, depressing, boring and lonely, leading to feelings of clinical depression, despair and abandonment. ${ }^{20}$ Indeed, loneliness, monotony and stigmatisation have been reported as frequently as potential positive aspects of segregation such as having time for reflection, which some patients find very therapeutic. ${ }^{21}$ In adult men diagnosed as HIV positive, social isolation is thought to be compounded by ruptures in relationships and the breakdown of social support networks. ${ }^{22}$ In addition, while there may be a high desire among patients to receive information and reassurance, being segregated appears to inhibit communication. ${ }^{18}{ }^{23}$ Colonisation with B cepacia has resulted in exclusion from CF conferences and support groups, leading to the loss of mutual support systems typically available to adults with $\mathrm{CF}^{24}$ and, consequently, to further increases in feelings of isolation, anger, and of being a "microbial leper". ${ }^{25}$ 


\section{INTERVENTION STRATEGIES}

Patients must attempt to reassemble coherent structures for social relationships if they are to adapt successfully to their new status. The most significant factors identified as being able to improve the experience for the patient are ones which enhance human interaction. While there is no doubt that the world wide web has become the greatest source of information and opened up a stream of new possibilities for facilitating communication between individuals with $\mathrm{CF}$, there are inherent pitfalls if such sources and processes are left unchecked. Web sites and chat rooms can, unfortunately, also be a fountainhead of disinformation, rumour and "folklore". As there are few resources currently available to develop such sites effectively, more immediate strategies must be found "beyond the Internet".

There are clear implications for how staff and family members can assist in ameliorating the psychosocial effects of isolation in hospital and social segregation..$^{20}$ Nursing staff and social workers, in particular, have key skills in working with patients in giving information or liaison capacities and have critical roles to play in the assessment of mood states and the provision of strategies aimed at improving the experience. ${ }^{21}$ While psychological therapists have the necessary skills to provide psychotherapy on an individual basis, such "reactive" strategies will only ever meet the needs of the few and such services tend to be under-resourced. It remains important to screen regularly for psychopathology, either by conventional or psychometric assessment, particularly when it is known that the patient has had previous psychological problems. Where indicated, psychotherapy must be sought. Psychological therapists can also adopt a more consultative role and help other team members to build on their skills-counselling or otherwise- and support them in supporting individuals who are segregated because of $B$ cepacia infection. This would not necessarily involve spending more time with people. On the contrary, it seems that the principal aim must be to empower patients, facilitate their selfcontrol, and to minimise boredom and rumination. Liaison with other professional groups-such as occupational therapists, who may instigate activity scheduling programmes-or agenciessuch as charities and action groups who may provide, among other things, befriending-will be of great benefit to patients. Other suggestions include improving the frequency and quality of written information and staff-patient communication and establishing regular visiting programmes. ${ }^{18}$

Patients themselves could also have important roles to play. Chronic disease self-management programmes $\left(\right.$ CDSM Ps) ${ }^{26} 27$ are small highly structured groups led by volunteers, all of whom have the condition, and focus on cognitive symptom management, exercise and nutrition, problem solving, and communication with medical professionals. In the UK the government is now actively supporting CDSM Ps with national pil ot schemes taking place over the next 3 years. ${ }^{28}$ Several CDSMPs already exist-for example, for patients with arthritis and multiple sclerosisand outcome studies have shown significant reductions in the severity of symptoms and pain, and gains in quality of life and resourcefulness. These studies also indicate the potential for further improving communication and doctorpatient relationships. ${ }^{29}$ Although difficult, it may be possible for small groups of patients with CF who are segregated because of $B$ cepacia and who have been genomovar typed to form CDSMPs. Where microbiological status and genomovar type are unknown, such groups could still take place using videoconferencing technology.

\section{CONCLUSIONS}

It is well recognised that being "hospitalised" has a negative effect on psychological functioning. Segregation and isolation may have even greater negative effects on emotional well being. Such patients have been shown to have significantly higher rates of anxiety and depression and significantly lower levels of self-esteem and control. They must reassemble coherent structures for social relationships if they are to adapt successfully to their new status. While the Internet offers improvements in communication and information dissemination, facilities for people with CF are generally unavailable and poorly monitored. M ore immediate solutions may rely on key members of CF teams, particularly nursing staff and social workers, facilitating patients' selfcontrol and empowerment and minimising boredom and rumination. Individual psychotherapy will benefit only the few patients whose emotional distress is identified and who are able to access direct psychological support. While such services remain in relative short supply, screening remains imperative. Psychological therapists working in CF teams may have a more consultative role to play in helping establish proactive intervention schemes. Specific nurse-led strategies, improvements in communication, visiting programmes and CDSM Ps should all be explored further.

With calls for segregation practices to be extended beyond patients with CF infected with B cepacia, there is an obligation to learn from the experiences of this group of patients who have already experienced radical changes to their medical management and support networks and to shape future management accordingly.

Correspondence to: Dr A J A Duff, Department of Clinical \& Health Psychology \& Regional CF Unit, St lames's University Hospital, Leeds LS9 7TF, UK; a.j.a.duff@leeds.ac.uk

Thorax 2002;57:756-758

\section{REFEREN CES}

1 Dodge JA, Morrison S, Lewis PA, et al. Cystic fibrosis in the United Kingdom, 1968-1995: incidence, population and survival. Arch Dis Child 1997:77:493-6.

2 Frederiksen $B$, Lanng $S$, Koch $C$, et al. Improved survival in the Danish centre treated cystic fibrosis patients: results of aggressive treatment. Pediatr Pulmonol 1996;21:153-8.

3 Ramsay BW. M anagement of pulmonary disease in patients with cystic fibrosis. $N$ Eng I Med 1996;335:179-88

4 Peckham D, Crouch S, Humphreys $\mathrm{H}$, et al. Effect of antibiotic treatment on inflammatory markers and lung function in cystic fibrosis patients with Pseudomonas cepacia. Thorax 1994;49:803-7.

5 Govan JWR. The Burkholderia cepacia complex: an update on epidemiology and host/ pathogen interactions. Paper presented at the 24th European Cystic Fibrosis Conference. Vienna, A ustria, 6-9 June 2001

6 Li Puma JJ, Dansen SE, N eilson DW, et al. Person to person transmission of Pseudomonas cepacia between patients with cystic fibrosis. Lancet 1990;336:1094-6.

7 UK CF Trust Infection Control Group. Burkholderia cepacia. Bromley, Kent: The CF Trust, 1999.

8 Smith DC, Gumery L, Smith EC, et al. Epidemic of Pseudomonas cepacia in an adult cystic fibrosis unit: evidence of person to persons transmission. J Clin Microbio 1993;31:3017-22

9 Jones AM, Govan JRW, Doherty CJ, et al. Spread of a multiresistant strain of Pseudomonas aeruginosa in an adult cystic fibrosis clinic. Lancet 2001;358:55 7-8.

10 McCallum SJ, Corkhill J, G allacher M, et al. Superinfection with a transmissible strain of Pseudomonas aeruginosa in adults with cystic fibrosis chronically colonised by $P$ aeruginosa Lancet 2001;358:558-60

11 Geddes D. O f isolates and isolation: Pseudomonas aeruginosa in adults with cystic fibrosis. Lancet 2001;358:522-3.

12 Blair C, Cull A, Freeman CP. Psychosocial functioning of young adults with cystic fibrosis and their families. Thorax 1994;49:798-802.

13 Duncan-Skingle F, Pankhurst FJ. A dults. In: Bluebond-Langner $M$, Lask $B$, Angst $D$, eds. Psychosocial aspects of cystic fibrosis. London: Arnold, 2001:161-71.

14 Thompson DR. A randomised controlled trial of inpatient nursing support for first time myocardial infarction patients and their partners: effects on anxiety and depression. J Adv N urs 1989;14:291-7.

15 Elliot D. M easuring patient anxiety in coronary care. A ust Crit Care 1992;5:12-6.

16 Teasdale $K$. The nurse's role in anxiety management. Prof N urse 1995;10:509-12.

17 Shuldham CM, Cunningham G, Hiscock M, et al. Assessment of anxiety in hospitalised patients. I Adv N urs 1995:22:87-93.

18 Ward D. Infection control: reducing the psychological effects of isolation. $\mathrm{Br} J \mathrm{~N}$ urs 2000;9:162-70

19 Gammon J. A nalysis of the stressful effects of hospitalisation and source isolation on coping and psychological constructs. Int J $N$ urs Pract 1998;4:84-96

20 Kelly-Rossini L, Perlman DC, Mason DJ. The experience of respiratory isolation for HIV-infected persons with tuberculosis. I Assoc $N$ urses AIDS Care 1996;7:29-36. 
21 Oldman $\mathrm{T}$. Isolated cases. $N$ urs Times 1998;94:67-70

22 Cherry K, Smith DH. Sometimes I cry. The experience of loneliness for men with AIDS. Health Comm 1993;5:181-208.

23 Campbell T. Feelings of oncology patients about being nursed in protective isolation as a consequence of cancer chemotherapy treatment. J Adv N urs 1999;30:439-47.

24 Waters S, Smith EG. Pseudomonas cepacia in cystic fibrosis: transmissibility and its implications. Lancet 1993;342:3-4.
25 Govan JRW, Brown PH, Maddison J. Evidence for transmission of Pseudomonas cepacia by social contact in cystic fibrosis. Lancet 1993;342:15-9.

26 Lorig K, Holman HR. Long-term outcomes of an arthritis self-management study: effects of reinforcement efforts. Soc Sci M ed 1989;3:217-26.

27 Lorig K, Mazonson PD, Holman HR. Evidence suggesting that health education for self-management in patients with chronic arthritis has sustained health benefits while reducing health care costs. Arthritis Rheum 1993;36:439-46.

28 Department of Health. The expert patient: a new approach to chronic disease

management for the 21 st century. London: Department of Health Expert Patients Task Force, 2001 (www.doh.gov.uk) healthinequalities).

29 Barlow JH. Self management literature review. Psychological Research Centre, University of Coventry (for the Department of Health Expert Patients Task Force), 2000.

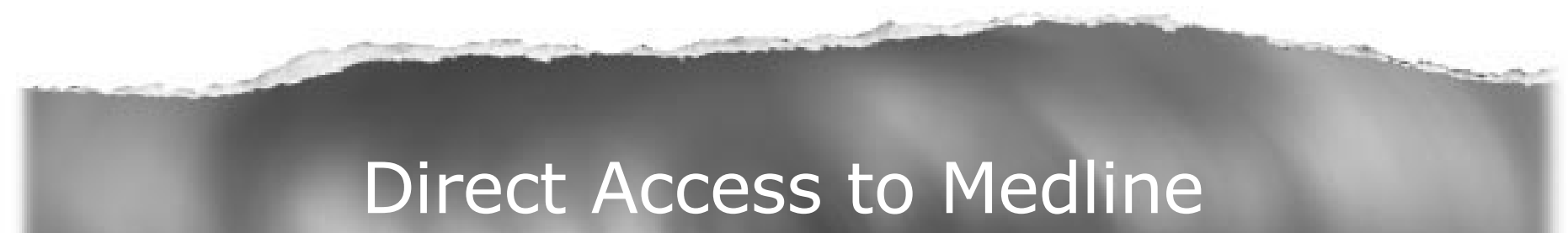

Medline

Link to Medline from the homepage and get straight into the National Library of Medicine's premier bibliographic database. Medline allows you to search across 9 million records of bibliographic citations and author abstracts from approximately 3,900 current biomedical journals.

www.thoraxjnl.com 